\title{
BMJ Global Health Diagnostic accuracy of neonatal foot length to identify preterm and low birthweight infants: a systematic review and meta-analysis
}

\author{
Lian V Folger, ${ }^{1}$ Pratik Panchal, ${ }^{2}$ Michelle Eglovitch, ${ }^{1}$ Rachel Whelan, ${ }^{1}$ Anne CC Lee
}

To cite: Folger LV, Panchal P, Eglovitch M, et al. Diagnostic accuracy of neonatal foot length to identify preterm and low birthweight infants: a systematic review and metaanalysis. BMJ Global Health 2020;5:e002976. doi:10.1136/ bmjgh-2020-002976

Handling editor Seye Abimbola

- Additional material is published online only. To view, please visit the journal online (http://dx.doi.org/10.1136/ bmjgh-2020-002976).

Received 23 May 2020 Revised 19 August 2020 Accepted 20 August 2020

Check for updates

(c) Author(s) (or their employer(s)) 2020. Re-use permitted under CC BY. Published by BMJ.

${ }^{1}$ Department of Pediatric Newborn Medicine; Global Advancement of Infants and Mothers (AIM) Lab, Brigham and Women's Hospital, Boston, Massachusetts, USA

${ }^{2}$ Translational Medicine, Gl Drug Discovery Unit, Takeda Pharmaceuticals, Cambridge, Massachusetts, USA

Correspondence to

Dr Anne CC Lee;

alee6@bwh.harvard.edu

\section{ABSTRACT}

Introduction Eighty percent of neonatal deaths occur among babies born preterm and/or small for gestational age (SGA). In sub-Saharan Africa and South Asia, approximately $40 \%$ of births occur outside of health facilities, and gestational age (GA) and birth weight are commonly unknown. Foot length (FL) has been proposed as a simple, surrogate measurement to identify and triage small babies born in the community. We conducted a systematic review and meta-analysis of the diagnostic accuracy of newborn $\mathrm{FL}$ to classify preterm and low birthweight infants.

Methods PubMed, EMBASE, Cochrane, Web of Science, POPLINE and WHO Global Health Library databases were searched. Studies of live-born infants that compared FL with GA and/or birth weight were included. Data on diagnostic accuracy were summarised, described, and pooled, as appropriate.

Results Six hundred and two studies were identified and 41 included. Techniques for measuring FL included use of a firm plastic ruler, callipers, footprint or a measuring board. Twelve studies assessed the diagnostic accuracy of $F \mathrm{~L}$ to identify preterm births; however, data were not pooled given heterogeneity and low quality of GA. 19 studies used FL to identify low birthweight infants ( $<2500 \mathrm{~g}$, $<2000 \mathrm{~g}$ ). Among studies in Asia ( $\mathrm{n}=3$ ), $\mathrm{FL} \leq 7.7 \mathrm{~cm}$ had pooled sensitivity and specificity of $87.6 \%(95 \% \mathrm{Cl} 61.1 \%$ to $99.0 \%)$ and $70.9 \%$ ( $95 \%$ Cl $23.5 \%$ to $95.1 \%$ ), respectively, to identify $<2500 \mathrm{~g}$ infants. FL $\leq 7.3 \mathrm{~cm}$ had $82.1 \%$ (95\% Cl $63.7 \%$ to $92.2 \%$ ) sensitivity and $82.1 \%(95 \% \mathrm{Cl} 59.2 \%$ to $90.8 \%)$ specificity for identifying $<2000 \mathrm{~g}$ infants $(\mathrm{n}=3)$. In the African studies $(\mathrm{n}=3), \mathrm{FL} \leq 7.9 \mathrm{~cm}$ had pooled sensitivity and specificity of $92.0 \%(95 \% \mathrm{Cl} 85.6 \%$ to $95.7 \%)$ and $71.9 \%(95 \% \mathrm{Cl} 44.5 \%$ to $89.1 \%$ ), respectively, to identify $<2500 \mathrm{~g}$ neonates.

Conclusions $\mathrm{FL}$ is a simple proxy measure that can identify babies of low birthweight with high sensitivity, though somewhat lower specificity. Additional research is needed to determine the validity of $\mathrm{FL}$ to identify preterm infants, and understand the programmatic impact of screening on healthcare seeking and outcomes.

PROSPERO registration number CRD42015020499

\section{INTRODUCTION}

Each year, an estimated 20.5 million newborns are born low birthweight (LBW) $\quad(<2500$ g) worldwide, with nearly three-quarters

\section{Key questions}

What is already known?

- An estimated $80 \%$ of neonatal deaths occur in small infants-either born preterm ( $<37$ weeks gestation) and/or small for gestational age.

- Gestational age and birthweight measurement are challenging and often not available among infants born outside of health facilities in low-income and middle-income settings.

- Neonatal foot length has been used as a simple, feasible surrogate method for identifying high-risk infants to link them with facilities and special newborn care in these settings.

What are the new findings?

- The measurement of gestational age among studies of foot length was heterogeneous, with generally low quality (ie. not early ultrasound).

- In pooled analysis of the Asian studies, foot length size of $<7.7 \mathrm{~cm}$ had $88 \%$ sensitivity and $71 \%$ specificity for identifying infants $<2500 \mathrm{~g}$, and foot length $\leq 7.3 \mathrm{~cm}$ had $82 \%$ sensitivity and $82 \%$ specificity for identifying $<2000 \mathrm{~g}$ infants.

- In pooled analysis of the African studies, foot length size of $\leq 7.9 \mathrm{~cm}$ had sensitivity and $92 \%$ and specificity of $72 \%$ to identify $<2500 \mathrm{~g}$ neonates.

What do the new findings imply?

- Measurement of foot length is a simple and lowcost screening tool with high sensitivity yet lower specificity to identify infants of low birthweight in community settings where birth weighing scales are unavailable.

- Additional research is needed to ascertain the validity of foot length to identify preterm infants, and to study the implementation and impact of programmes to identify and manage low birthweight infants at the community level.

occurring in South Asia and sub-Saharan Africa. ${ }^{1}$ LBW may result from preterm birth and/or small for gestational age (SGA), commonly defined as birth weight below the $10 \%$ for gestational age and sex. 
Complications of preterm birth are now the leading cause of mortality among children under 5 , resulting in 1 million deaths annually. ${ }^{2}$ Small size at birth, due to either preterm birth or SGA, accounts for more than $80 \%$ of neonatal deaths worldwide. ${ }^{3}$ Thus, increasing attention has focused on the identification, triage and management of small babies in low-income and middle-income countries (LMIC) to reduce neonatal morbidity and mortality.

In sub-Saharan Africa and South Asia, approximately $40 \%$ of births occur without skilled birth attendants, and, although facility births are increasing, 30\%-45\% of births still occur in the community setting-and as high as $65 \%$ among women from rural areas and the poorest wealth quintile. ${ }^{4}$ The identification of preterm and SGA babies in these settings is challenging. Many babies in LMIC are not weighed at birth, ${ }^{1}$ both in home births and in primary health facilities where weighing scales are not available and/or staff are overburdened. In 2015, 40 million (one-third) of babies born globally had no recorded birthweight, $97 \%$ of whom were in Asia and Africa. ${ }^{5}$ Furthermore, in the majority of settings in LMIC, GA of the pregnancy is often uncertain or unavailable. ${ }^{6}$ Last menstrual period may be unknown or affected by poor recall, ${ }^{78}$ and ultrasonography is not available, or is only available late in pregnancy, when traditional ultrasound has been less accurate for GA dating. ${ }^{7-9}$

The early and accurate identification of small and preterm infants in these settings is the first step to providing these high-risk babies with potentially life-saving interventions. This was recognised as a global priority in The Global Action Report on Preterm Birth-a collaboration between March of Dimes, the Partnership for Maternal, Newborn and Child Health, Save the Children and the WHO. ${ }^{10}$ Thus, it is critical to identify accurate screening techniques that are simple, low cost and feasible, which could be adopted in LMIC settings. Surrogate neonatal anthropometric measures, such as mid-upper arm, chest and head circumferences, have been tested for identifying LBW and/or preterm newborns. ${ }^{11-13}$ For this review, we chose to focus on neonatal foot length, which has emerged as a programmatically useful method to identify small babies in LMIC. Measurement of the foot can be done with locally available, portable, low-cost tools; does not require heavy or specialised equipment (eg, weighing scales or circumference tape measures); and the foot is easy to access without requiring undressing or unwrapping the baby.

The aim of this study was to systematically review the evidence for the diagnostic accuracy of foot length as a measure to identify high-risk preterm and LBW babies.

\section{METHODS \\ Search strategy}

We conducted a systematic review of the published literature. The searches were initially performed in May 2015 and updated in May 2020 (figure 1). The Preferred Reporting Items for Systematic Reviews and MetaAnalyses $^{14}$ (PRISMA) statement and review protocol are available in the online supplemental appendix (online supplemental web appendix 1-2). The following databases were searched: PubMed, Embase, Cochrane, Web of Science, Popline and the WHO Global Health Libraries/regional databases (Latin American and Caribbean Health Sciences Literature (LILACS), Index Medicus for South East Asia Region (IMSEAR), Western Pacific Regional Office (WPRO), Index Medicus for the Eastern Mediterranean Region (IMEMR), Africa Index Medicus (AIM). Detailed search terms are available in online supplemental web appendix 3 .

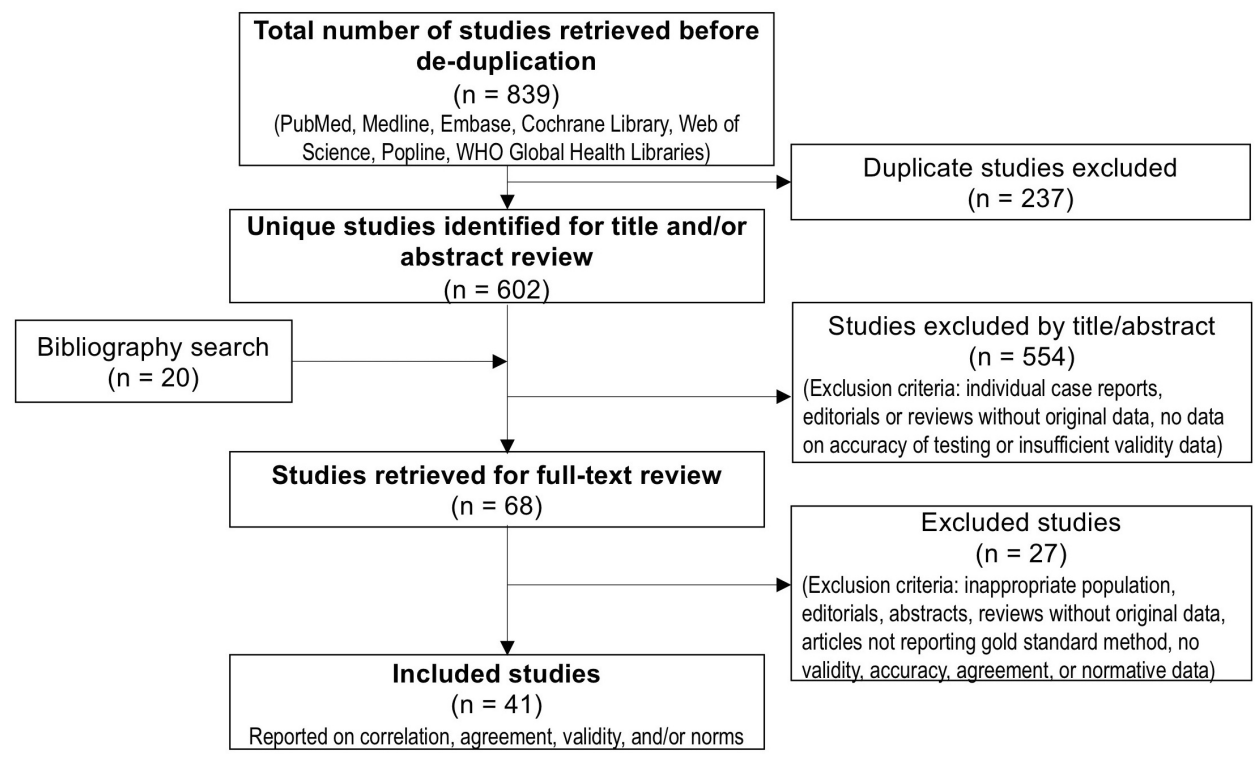

Figure 1 Foot length flow diagram. Diagram of the screening process to identify studies for inclusion in foot length review; adapted from Moher et al $\left.^{14}\right)$. 


\section{Inclusion criteria}

There were no language restrictions. Non-English abstracts were translated to English to determine if they met inclusion criteria, and relevant full-text articles were then translated to English using Google Translate or a fluent speaker.

Articles were considered for inclusion if the study: (1) included live-born neonates, (2) included data on newborn foot length and either GA or birthweight and (3) reported at least one statistic comparing foot length with GA or birth weight or reported on inter-rater or intra-rater reliability. Preterm births were defined as liveborn infants born at $<37$ weeks gestation. We included data on validity for the identification of preterm infants, infants $<2500 \mathrm{~g}$ (LBW), and $<2000 \mathrm{~g}$.

\section{Exclusion criteria}

We excluded individual case reports, duplicate studies and studies of specialised subpopulations or selected populations. We also excluded studies that reported on fetal deaths or ultrasound-measured/fetal foot length.

\section{Data extraction}

Data were extracted on study setting, design, population characteristics, participant selection, foot length measurement technique, GA or birth weight measurement, correlation, inter-rater and intra-rater reliability, and validity of foot length to identify preterm and/or LBW infants ( $<2500 \mathrm{~g}$ and/or $<2000 \mathrm{~g}$ ) (online supplemental web appendix 2). Two independent reviewers conducted data extraction, and differences were resolved by a third reviewer. For studies reporting diagnostic accuracy, data on the validity of all reported or published foot length cut-offs were extracted. One study reported vertical foot length distance, ${ }^{15}$ and this was converted to an equivalent heel-hallux distance using methods reported in online supplemental web appendix 4.

\section{Study quality assessment}

For studies reporting diagnostic accuracy, methodological quality was assessed per the Cochrane Diagnostic Test Accuracy working group recommendations using the Quality Assessment of Diagnostic-Accuracy Studies (QUADAS-2) ${ }^{16}$ which was modified to fit the context of this study (online supplemental web appendix 2, section 5). Individual studies were evaluated for limitations and biases in the following five domains: study design, population selection and representativeness, definitions, precision and generalisability to the population of interest. The study-level QUADAS-2 score sheets are available on request.

\section{Statistical analysis}

Stata V.15 (StataCorp) was used for data analysis. Studies were summarised and stratified for analysis by major WHO geographical region (ie, Asia, Africa). The regional grouping is based on the approach used by the Child Health Epidemiology Reference Group ${ }^{17}$ and hypothesised differences in birth size and foot length cut-offs between regions. Studies with comparable foot length thresholds were grouped. Studies reported results differently with respect to inclusion of the specific cut-off value (ie, $<$ or $\leq$ the threshold). For the practicality of health worker interpretation and future implementation, we grouped studies with cutoffs as less than or equal to $(\leq)$ a particular foot length cut-off. For example, assuming a precision of $0.1 \mathrm{~cm}(1 \mathrm{~mm})$, we grouped studies that analysed a $\leq 7.4 \mathrm{~cm}$ cut-off together with those that used $\mathrm{a}<7.5 \mathrm{~cm}$ cut-off.

Outcomes of interest for pooled diagnostic accuracy analysis included preterm birth, birthweight $<2500 \mathrm{~g}$ and $<2000$ g. For pooled analysis with adequate study numbers, hierarchal models were used to pool sensitivity and specificity using the STATA metandi command. For those with $<4$ studies, proportions were logit transformed and standard errors calculated. Meta-analysis was conducted with random effects using the method of DerSimonian and Laird. ${ }^{18}$

\section{Patient and public involvement}

This study did not involve any patients.

\section{RESULTS}

Six hundred and two unique studies were identified in the searches, and 68 full-text studies were reviewed. Forty-one studies were extracted and included in the final review (figure 1). Among these, 19 studies reported diagnostic accuracy data. Nine studies reported data on the diagnostic accuracy of foot length for identifying both preterm births and LBW infants $(<2500 \mathrm{~g}$ or $<2000 \mathrm{~g}), 3$ papers reported only on preterm, and 7 papers reported only on LBW. The remaining papers $(n=22)$ reported either data on the correlation between foot length and GA or birth weight, normal values of foot length for different GAs, and/or inter-rater or intra-rater reliability.

\section{Overall study characteristics}

The basic study characteristics of all included studies are shown in online supplemental web appendix 5 . Thirty-five were in LMIC (25 in Asia, 10 in sub-Saharan Africa), with the remainder in high-income countries (3 in Europe, 3 in North America). Twenty-nine studies were conducted in health facilities/hospitals, seven were community based and the rest were not specified.

\section{Study quality}

The overall QUADAS-2 summary figure for all included studies with diagnostic accuracy data $(n=19)$ is shown in online supplemental web appendix 6 . In general, the quality of the studies was relatively low. There was a high risk of bias in over half of the studies related to patient selection and reference standard. Many studies were conducted in neonatal intensive care units (NICUs), which may affect the generalisability of the screening tool's diagnostic accuracy in the general population of newborns. Risk of bias from the reference standard was also high in most studies assessing foot length and GA, 
Table 1 Foot length measurement methods

\begin{tabular}{|c|c|}
\hline \multicolumn{2}{|l|}{ Measurement tools } \\
\hline $\begin{array}{l}\text { Firm ruler (plastic, metal, } \\
\text { wooden) (See online } \\
\text { supplemental WebAppendix 7a) }\end{array}$ & Firm; low cost and locally accessible; does not required specialised tool or procurement. \\
\hline Sliding callipers & Precise however requires specialised tool, more costly and difficult to procurethan ruler. \\
\hline Flexible measuring tape & $\begin{array}{l}\text { Low cost, locally available. Flexible tape is not fixed or firm; may be less reliable and prone } \\
\text { to variation between measurements. }\end{array}$ \\
\hline $\begin{array}{l}\text { Footprint (See online } \\
\text { supplemental WebAppendix } 4 a \text { : } \\
\text { eFigure 1) }\end{array}$ & $\begin{array}{l}\text { Requires firm surface. Can be measured retrospectively. Challenges include local/cultural } \\
\text { beliefs regarding foot/finger prints and requires cleaning foot afterwards. }\end{array}$ \\
\hline $\begin{array}{l}\text { Foot length measuring board } \\
\text { (See online supplemental } \\
\text { WebAppendix } 7 \mathrm{~b} \text { ) }\end{array}$ & $\begin{array}{l}\text { Precise, reliable; however, requires specialised tool that maybe difficult to manufacture or } \\
\text { procure. }\end{array}$ \\
\hline \multicolumn{2}{|l|}{ Measurement Techniques/Axis } \\
\hline Heel-to-hallux & Linear distance measured from the base of the heel to the tip of hallux (big toe) \\
\hline Heel-to-longest toe & $\begin{array}{l}\text { Linear distance measured from the base of the heel to the tip of the longest toe (first, } \\
\text { second, or third digit) }\end{array}$ \\
\hline Vertical distance & $\begin{array}{l}\text { Linear distance measured from the base of the heel to longest digit, along vertical axis of } \\
\text { foot }\end{array}$ \\
\hline
\end{tabular}

given that only two studies had an early ultrasound-based reference, with the remaining using a clinical newborn examination or last menstrual period. The individual study QUADAS-2 data are available on request.

\section{Foot length measurement techniques}

Among the studies identified, investigators reported several different techniques and tools for measuring foot length (table 1; online supplemental web appendix 7). The physical distances measured included: (1) the maximal heel-to-hallux distance (base of the heel to tip of the hallux), (2) distance from base of the heel to the tip of the longest digit, and (3) maximal perpendicular vertical distance. Measurement tools included a firm ruler, sliding callipers, flexible measuring tape, a foot length measuring board, and, lastly, foot print and post hoc measurement on paper.

\section{Normal distribution of foot length by GA}

Nine studies were identified that reported the normal distribution of foot length by GA, which are shown in table 2 by region. Of those, six were from Asia, ${ }^{19-24}$ two from Europe, ${ }^{25} 26$ and one from North America. ${ }^{27}$

\section{Correlation of foot length with GA and birth weight}

Seventeen studies reported on the correlation of foot length with GA, with correlation coefficients ranging from 0.093 to 0.99 (median $0.873 ; \mathrm{n}=14$ studies) (online supplemental web appendix 8A). One of the two studies that had an ultrasound-based reference reported the lowest correlation coefficient (0.093), though included a narrow range of GA. ${ }^{28}$ The majority of studies $(n=13)$ with GA data used neonatal clinical assessment as the reference standard, eight of which reported correlation coefficients greater than 0.85 .

Twenty-one studies reported on the correlation between birthweight and foot length (13 Asia, 6 Africa, 1 Europe) (online supplemental web appendix 8B). Correlation coefficients ranged from 0.213 to 0.951 (median 0.755; $\mathrm{n}=14$ studies). Data were not pooled on correlation coefficients due to the lack of reporting on the type of correlation coefficient (ie, Spearman vs Pearson) for a majority of studies.

\section{Diagnostic accuracy of foot length to identify preterm births}

We identified 12 studies that assessed the diagnostic accuracy of foot length to classify preterm infants (Asia $n=8$, Africa n=4) (table 3). Eight studies reported areas under the curve (AUCs) for identifying infants $<37$ weeks, which ranged from 0.52 to 0.89 in 5 South Asian studies, ${ }^{12} 28-31$ and from 0.86 to 0.95 in 3 African studies ${ }^{32-34}$ (table 3). The eight Asian studies used different methods of reference standard GA determination. Five studies used a postnatal clinical exam (New Ballard Score, NBS) as the reference standard, and reported relatively high diagnostic accuracy. ${ }^{12} 23313536$ On the other hand, the three Asian studies that used an LMP or ultrasound-based reference standard GA reported lower sensitivity and specificity. ${ }^{28-30}$ Similarly, three studies from Africa used a postnatal clinical exam (NBS and/or Eregie) as the GA reference standard, and reported relatively higher diagnostic accuracy. ${ }^{32} 3437$ One 2019 study conducted in rural Tanzania, which used an ultrasound-based reference standard GA, reported comparably high diagnostic accuracy $^{33}$ - higher than that of the one other study (in Bangladesh) that had ultrasound dating. ${ }^{28}$ 
Table 2 Normative foot length data by gestational age (GA)

\begin{tabular}{|c|c|c|c|c|c|c|}
\hline \multirow[b]{2}{*}{ Author (year) } & \multirow[b]{2}{*}{ Country } & \multirow[b]{2}{*}{ GA reference standard } & \multirow{2}{*}{$\begin{array}{l}\text { Foot length } \\
\text { distance } \\
\text { measured }\end{array}$} & \multicolumn{3}{|c|}{ Mean foot length for GA, cm (SD) } \\
\hline & & & & 28 weeks & 34 weeks & 37 weeks \\
\hline \multicolumn{7}{|l|}{ Asia } \\
\hline Kulkarni²1 (1992) & India & LMP and Dubowitz score & Heel-to-hallux & $5.66(0.68)$ & $6.70(0.64)$ & $6.99(0.56)$ \\
\hline $\mathrm{Kabra}^{20}(1989)$ & India & LMP and Dubowitz score & Heel-to-hallux & $5.28(0.70)$ & $6.83(0.38)$ & $7.55(0.54)$ \\
\hline Mathur ${ }^{22}$ (1984) & India & LMP & Heel-to-hallux & $5.69(0.35)$ & $6.93(0.44)$ & $7.26(0.34)$ \\
\hline Singhal $^{23}(2014)$ & India & $\begin{array}{l}\text { LMP and Extended New } \\
\text { Ballard Score }\end{array}$ & $\begin{array}{l}\text { Heel-to- } \\
\text { longest toe }\end{array}$ & $5.45(0.26)$ & $6.80(0.21)$ & $7.53(0.28)$ \\
\hline Srivastava $^{24}$ (2015) & India & $\begin{array}{l}\text { Extended New Ballard } \\
\text { Score }\end{array}$ & $\begin{array}{l}\text { Heel-to- } \\
\text { longest toe }\end{array}$ & $5.50(0)$ & $6.54(0.24)$ & $7.45(0.13)$ \\
\hline Rakkappan $^{19}$ (2016) & India & - & - & 5.26 & 6.11 & - \\
\hline \multicolumn{7}{|l|}{ Europe } \\
\hline Merlob $^{25}$ (1984) & Israel & $\begin{array}{l}\text { LMP, Dubowitz score, and } \\
\text { anterior vascular capsule of } \\
\text { the lens examination }\end{array}$ & Heel-to-hallux & $5.25(0.53)$ & $6.81(0.70)$ & $7.53(0.60)$ \\
\hline Vocel $^{26}(1978)$ & Czechoslovakia & LMP & Heel-to-hallux & - & $6.83(0.31)$ & $7.23(0.50)$ \\
\hline \multicolumn{7}{|l|}{ Americas } \\
\hline Usher $^{27}$ (1969) & Canada & LMP & $\begin{array}{l}\text { Heel-to- } \\
\text { longest toe }\end{array}$ & $5.54(0.31)$ & $6.96(0.38)$ & $7.80(0.39)$ \\
\hline
\end{tabular}

(-) symbol indicates that data is not available for that paper.

LMP, last menstrual period.

\section{Diagnostic accuracy of foot length to identify LBW infants $(<2500 \mathrm{~g})$}

We identified 15 cohorts in which the diagnostic accuracy of foot length to identify $<2500 \mathrm{~g}$ infants was assessed (Asia $n=8$, Africa $n=7$ ) (online supplemental web appendices 9, 10A,B). Seven reported AUCs, which ranged from 0.84 to 0.94 in the two Asian studies, ${ }^{12} 15$ and 0.74 to 0.97 in the five African studies ${ }^{32-34} 3839$ (online supplemental web appendix 9).

Diagnostic accuracy data were pooled for the identification of infants $<2500 \mathrm{~g}$ for several cutoffs (table 4 ). Among the Asian studies, a foot length cut-off of $\leq 7.7$ $\mathrm{cm}$ had a pooled sensitivity of $87.6 \%(95 \%$ CI $55.7 \%$ to $97.5 \%)$ and specificity of $70.9 \%$ (95\% CI $23.5 \%$ to $95.1 \%)$ ( $\mathrm{n}=3$ studies) (table 4). ${ }^{153540}$ Among the African studies, the pooled sensitivity and specificity for a foot length cut-off of $\leq 7.9 \mathrm{~cm}$ were $92.0 \%$ (95\% CI $85.6 \%$ to $95.7 \%$ ) and $71.9 \%(95 \%$ CI $44.5 \%$ to $89.1 \%)$, respectively $(\mathrm{n}=3$ studies) (table 4). ${ }^{32} 3741$

Diagnostic accuracy of foot length to identify infants $<\mathbf{2 0 0 0} \mathbf{g}$ Four studies reported on the diagnostic accuracy of foot length to identify infants $<2000 \mathrm{~g}$ in Asia (online supplemental web appendix 10C). ${ }^{15293040}$ Two studies reported AUCs (0.88 and 0.93, respectively) (online supplemental web appendix 9). ${ }^{1529}$ In the meta-analysis, a foot length cut-off of $\leq 7.3 \mathrm{~cm}$ classified $<2000 \mathrm{~g}$ infants (table 4) with sensitivity of $82.1 \%(95 \%$ CI $63.7 \%$ to $92.2 \%)$ and specificity of $82.1 \%$ (95\% CI $59.2 \%$ to $90.8 \%) \quad(n=3$ studies). 152940

\section{Inter- and intra-rater reliability}

Eight studies were identified that compared the agreement of repeated foot length measurements between and/ or within assessors (online supplemental web appendix 11). Five studies were conducted in Asia, ${ }^{15} 35$ 42-44 two in Africa, ${ }^{4546}$ and one in Europe. ${ }^{47}$ Four studies reported on interobserver kappa to classify small feet, which ranged widely from 0.30 to $0.82 . .^{35-46}$ Of the eight studies, the majority $(n=5)$ were in hospital settings. Measurements were conducted by medical staff (physicians, nurses, midwives) in three studies, ${ }^{35} 4547$ while another three studies included measurements by community volunteers, caretakers or field workers ${ }^{15} 4446$ (not reported in two studies). Three studies were in community settings, and two compared foot length measured by a healthcare worker to that of a community volunteer or caretaker, reporting kappa statistics of 0.53 and $0.82 .{ }^{44} 46$ One study in Tanzania found that community volunteers systematically undermeasured foot length by a mean of 0.26 cm compared with researchers. ${ }^{46}$ Four studies reported on intra-rater reliability, with coefficients of variation ranging from $1.05 \%{ }^{47}$ to $1.56 \% .^{42}$ Another study reported a within-infant range of measures of $\leq 0.2 \mathrm{~cm}$ in $98.4 \%$ of infants. $^{15}$

\section{DISCUSSION}

Improving the identification and care of small, high-risk babies is essential to reduce the global burden of neonatal morbidity and mortality. Given that GA and birth weight 


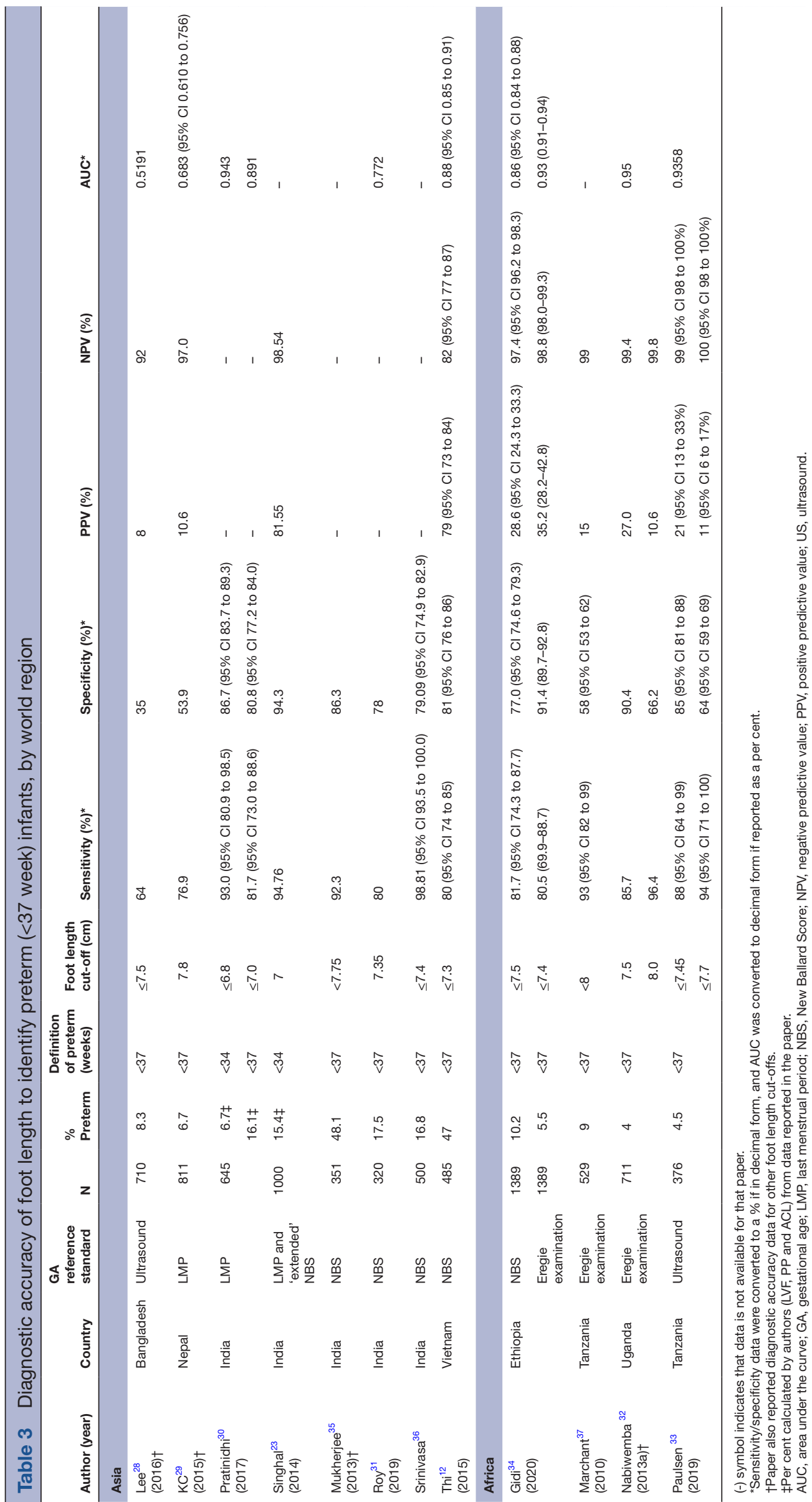

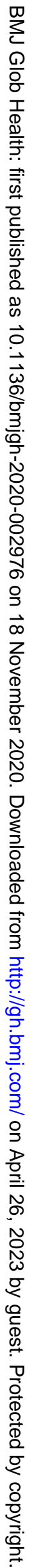


Table 4 Pooled sensitivity and specificity for all available foot length thresholds to identify low birthweight neonates $(<2500 \mathrm{~g}$ and $<2000 \mathrm{~g})$

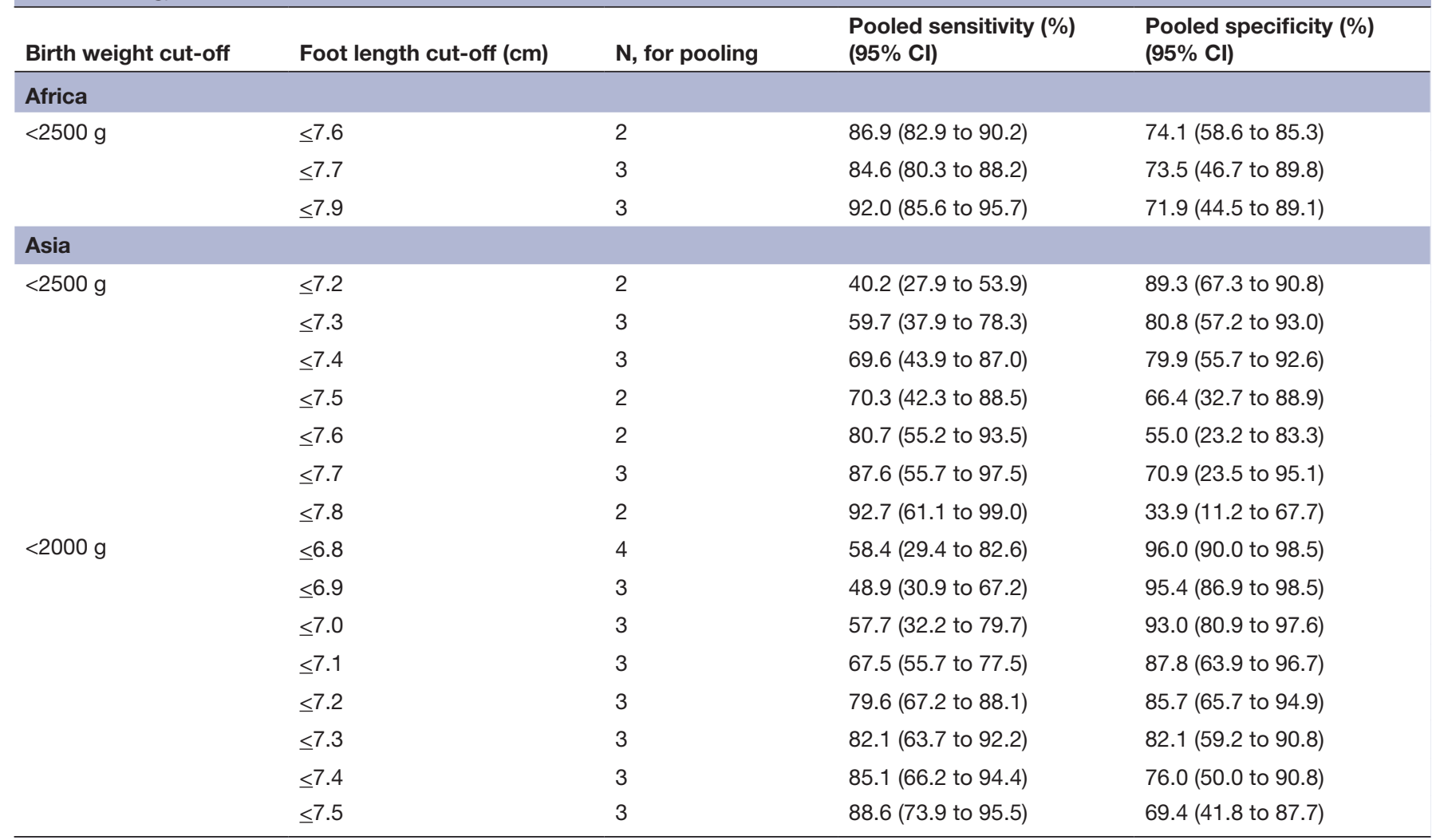

information is commonly missing in half of births in subSaharan Africa and South Asia, ${ }^{16}$ foot length measurement has emerged as a promising method to identify vulnerable infants born in community settings. In this systematic review and meta-analysis, we found that foot length thresholds of $\leq 7.7 \mathrm{~cm}$ in Asia and $\leq 7.9 \mathrm{~cm}$ in Africa classified LBW $(<2500 \mathrm{~g})$ infants with high sensitivity and lower specificity, and foot length $\leq 7.3 \mathrm{~cm}$ had relatively high sensitivity and specificity $(>80 \%)$ to classify infants $<2000 \mathrm{~g}$. Data assessing the accuracy of foot length for identifying preterm infants were limited by both quality and heterogeneity of reference standard GA dating method.

Different methods of foot length measurement have been described in the literature. Some investigators have used specialised or higher cost equipment, such as customised measuring boards ${ }^{15224247}$ or callipers. ${ }^{11192148}$ The most common method used across studies was the measurement of the heel-to-hallux (or to longest digit) with a firm ruler. This method is low cost, easy to train and feasible at the community level. Two studies compared the diagnostic accuracy of different measuring techniques (firm plastic ruler, measuring tape, footprint), and both found that the firm ruler had the highest predictive score for identifying both preterm and LBW newborns. ${ }^{29} 32$ Feasibility, training, standardisation and cost of equipment are key considerations for scalability in LMIC. In particular, standardising the landmarks used in foot length measurement is critical. The majority of studies that reported normative values for foot growth used the maximum heel-hallux distance, ${ }^{20-22} 2526$ though several used the heel-to-longest toe distance. ${ }^{2324} 27$ Having standardised landmarks for the distance measured is essential for both consistency of foot length measurements and comparisons between populations.

In the studies included in this review, data on the accuracy of foot length to identify preterm births were heterogeneous and generally of low quality. Only two studies used an early ultrasound-based reference standard GA, ${ }^{28} 33$ while most relied on clinical exam to determine GA, which estimates GA within \pm 4 weeks of ultrasound dating. ${ }^{49}$ The Eregie examination was commonly used in Africa. In this simplified examination, newborn anthropometrics (head circumference and mid-upper arm circumference) are included, and thus, dating is strongly influenced by newborn size. ${ }^{50}$ In a systematic review, out of three studies assessing the diagnostic accuracy of the Eregie examination, only one used an ultrasound reference and found that the Eregie dated pregnancies within \pm 3.5 weeks of ultrasound dating. ${ }^{28} 49$ In this review, among the Asian studies, the range of diagnostic accuracy ranged widely (sensitivity: 64\%-98\%, specificity: $35 \%-94 \%$ ), which may be due to the variation of reference standard GA dating methods, or potentially due to the challenge of discriminating SGA versus preterm infants in settings with high prevalence of fetal growth restriction. In South Asia, this prevalence is as high as $30 \%$. In addition, neonatal clinical examinations (used to determine reference GA in five of the eight Asian 
studies) have been shown to systematically underestimate GA among growth-restricted infants. ${ }^{49}$

Foot length was a reasonable proxy of infant size to identify LBW infants. The foot length thresholds to classify LBW were lower in Asia, where babies are smaller and SGA is more prevalent. ${ }^{151}$ For studies in Asia, a foot length cut-off of $\leq 7.7 \mathrm{~cm}$ identified $<2500 \mathrm{~g}$ infants with pooled sensitivity of $87.6 \%$ and specificity of $70.9 \%$; for identifying $<2000 \mathrm{~g}$ infants in Asia, a foot length cut-off of $\leq 7.3 \mathrm{~cm}$ had $82.1 \%$ sensitivity and $82.1 \%$ specificity. In Africa, a foot length cutoff of $\leq 7.9 \mathrm{~cm}$ had a pooled sensitivity of $92.0 \%$ and specificity of $71.9 \%$ to identify $<2500 \mathrm{~g}$ infants. The balance of sensitivity and specificity is a critical consideration in health systems that must weigh the increasing demand generated by identifying and referring more high-risk babies with the supply of available services and the risk of overburdening health systems. Based on our data, if a community-based foot length screening programme was implemented to refer LBW $(<2500 \mathrm{~g})$ infants in South Asia, where the prevalence of LBW is $30 \%$, in a population of 100000 newborns, there would be 26400 LBW infants correctly identified, 3600 LBW babies missed and 20300 non-LBW babies who were over-referred (false positives). Approximately $57 \%$ referred to health facilities would be truly LBW, and $93 \%$ of babies with foot length $>7.8 \mathrm{~cm}$ would not be LBW. In sub-Saharan Africa, where the prevalence of LBW $(<2500 \mathrm{~g})$ is $16.4 \%$, ${ }^{1}$ in a population of 100000 newborns, 15088 LBW babies would be correctly identified, 1312 LBW babies would be missed and 23408 over-referred. Approximately $40 \%$ of referred babies would be LBW, and $98 \%$ of babies with foot length $>8.0 \mathrm{~cm}$ would not be LBW. The local health system and public health implications should be considered for the implementation of any such screening programmes.

Training and standardisation are important considerations for programmatic implementation in LMIC. Intrarater and inter-rater agreement was generally high for neonatal foot length measurement. Foot length measurement is advantageous, as it can be easily performed with minimal medical training. Two studies assessed inter-rater agreement between a healthcare provider or researcher and a lay community health worker or caretaker, a comparison of important programmatic relevance. ${ }^{446}$ In Tanzania, Marchant et al reported that community volunteers systematically undermeasured foot length compared with research staff and overestimated those needing special care in the community. ${ }^{46}$ Reliability and continued quality assurance of measurements are important considerations for the potential scale up of this tool in programmatic and research settings, especially considering reliability in a variety of users.

There are several important limitations to this review. The overall quality of studies included in the review was low, with limitations in the quality of reference standard GA data, reporting and selection bias. There is a need for more studies with high-quality ultrasound dating or best obstetric estimate as the reference standard. In addition, we did not put a date restriction on our studies, as many of the original foot length articles were published in the 1970s. However, all studies that reported diagnostic accuracy data and were included in the meta-analyses were from after 2000, with the majority published after 2010 . We conducted pooled analysis by major WHO world region, though countries within these regions are heterogeneous and optimal foot length cutoffs may vary by country. Finally, we limited the scope of this review to diagnostic accuracy only, and it would be valuable to assess the effect of foot length measurement as a screening tool on referrals, care seeking behaviours and infant health outcomes. We are aware of an upcoming study in $\mathrm{Nepal}^{52}$ that will assess these outcomes.

\section{CONCLUSIONS}

In summary, improving the identification of small babies at the community level is a critical first step to triage these high-risk infants and provide timely and potentially lifesaving interventions. Foot length is a low-cost, simple and feasible measurement, with potential to identify LBW infants in low-resource communities. Standardisation of landmarks and measurement techniques is important. More studies are needed with accurate GA dating to determine the diagnostic accuracy of foot length measurement as a screening tool to identify preterm infants in LMIC. Given the lower specificity and potential for over-referral, alternative surrogate measures with higher specificity should also be sought and studied. Finally, programmatic and implementation research is needed to determine the effect of such screening programmes on newborn care seeking and health outcomes.

Acknowledgements We would like to acknowledge the students who were also part of the GA working group in the BWH Global AIM Lab (Lauren Schaeffer, Chelsea Clark). We would also like to thank the BWH Department of Pediatric Newborn Medicine and Terrie Inder for their support of this work.

Contributors LVF coordinated and conducted screening and data extraction, assisted with designing search strings and data analysis, codrafted and revised the manuscript, and approved the final manuscript as submitted. PP designed the database searches, carried out initial screening and data extraction, and performed data analysis. He reviewed the manuscript, and approved the final manuscript as submitted. ME screened and extracted data, summarised data, reviewed and revised the manuscript, and approved the final manuscript as submitted. RW coordinated and supervised initial screening and data extraction, reviewed the manuscript, and approved the final manuscript as submitted. ACCL conceptualised and designed the study, coordinated and supervised data collection, completed secondary data extraction, conducted meta-analyses and codrafted and finalised the manuscript, and approved of the manuscript as submitted.

Funding This work was supported by the Bill \& Melinda Gates Foundation through grant OPP1130198.

Competing interests None declared.

Patient and public involvement Patients and/or the public were not involved in the design, or conduct, or reporting, or dissemination plans of this research.

Patient consent for publication Not required.

Provenance and peer review Not commissioned; externally peer reviewed.

Data availability statement Data are available on request from the corresponding author.

Open access This is an open access article distributed in accordance with the Creative Commons Attribution 4.0 Unported (CC BY 4.0) license, which permits others to copy, redistribute, remix, transform and build upon this work for any purpose, provided the original work is properly cited, a link to the licence is given, and indication of whether changes were made. See: https://creativecommons.org/ licenses/by/4.0/. 


\section{REFERENCES}

1 Blencowe H, Krasevec J, de Onis M, et al. National, regional, and worldwide estimates of low birthweight in 2015, with trends from 2000: a systematic analysis. Lancet Glob Health 2019;7:e849-60.

2 Liu L, Oza S, Hogan D, et al. Global, regional, and national causes of under-5 mortality in 2000-15: an updated systematic analysis with implications for the sustainable development goals. Lancet 2016;388:3027-35.

3 Lawn JE, Blencowe H, Oza S, et al. Every newborn: progress, priorities, and potential beyond survival. Lancet 2014;384:189-205.

4 UNICEF. Delivery care. UNICEF data: monitoring the situation of children and women, 2020. Available: https://data.unicef.org/topic/ maternal-health/delivery-care/ [Accessed 20 May 2020].

5 UNICEF, WHO. UNICEF-WHO low birthweight estimates: levels and trends 2000-2015. Geneva: United Nations Children's Fund (UNICEF), World Health Organization (WHO), 2019.

6 Chawanpaiboon S, Vogel JP, Moller A-B, et al. Global, regional, and national estimates of levels of preterm birth in 2014: a systematic review and modelling analysis. Lancet Glob Health 2019;7:e37-46.

7 Aliyu LD, Kurjak A, Wataganara T, et al. Ultrasound in Africa: what can really be done? J Perinat Med 2016;44:119-23.

8 Blencowe $\mathrm{H}$, Cousens S, Chou D, et al. Born too soon: the global epidemiology of 15 million preterm births. Reprod Health 2013;10 Suppl 1:S2.

9 Committee on Obstetric Practice, the American Institute of Ultrasound in Medicine, and the Society for Maternal-Fetal Medicine. Committee opinion no 700: methods for estimating the due date. Obstet Gynecol 2017;129:e150-4.

10 Born Too Soon. The Global Action Report on Preterm Birth. In: Howson CP KM, Lawn JE, eds. March of Dimes, the partnership for maternal, newborn, and child health $(P M N C H)$, save the children. Geneva, Switzerland: World Health Organization (WHO), 2012.

11 Thawani R, Dewan P, Faridi MMA, et al. Estimation of gestational age, using neonatal anthropometry: a cross-sectional study in India. $\checkmark$ Health Popul Nutr 2013;31:523-30.

12 Thi HN, Khanh DKT, Thu HLT, et al. Foot length, chest circumference, and mid upper arm circumference are good predictors of low birth weight and prematurity in ethnic minority newborns in Vietnam: a hospital-based observational study. PLoS One 2015;10:e0142420.

13 Goto E. Meta-Analysis: identification of low birthweight by other anthropometric measurements at birth in developing countries. $J$ Epidemiol 2011;21:354-62.

14 Moher D, Liberati A, Tetzlaff J, et al. Preferred reporting items for systematic reviews and meta-analyses: the PRISMA statement. BMJ 2009;339:b2535.

15 Mullany LC, Darmstadt GL, Khatry SK, et al. Relationship between the surrogate anthropometric measures, foot length and chest circumference and birth weight among newborns of Sarlahi, Nepal. Eur J Clin Nutr 2007;61:40-6.

16 Whiting PF, Rutjes AWS, Westwood ME, et al. QUADAS-2: a revised tool for the quality assessment of diagnostic accuracy studies. Ann Intern Med 2011;155:529-36.

17 Katz J, Lee AC, Kozuki N, et al. Mortality risk in preterm and small-for-gestational-age infants in low-income and middle-income countries: a pooled country analysis. Lancet 2013;382:417-25.

18 DerSimonian R, Laird N. Meta-Analysis in clinical trials. Control Clin Trials 1986;7:177-88.

19 Rakkappan I, Kuppusamy N. Newborn foot length measurement to identify high-risk neonate. Int J Sci 2016;4:13-19.

20 Kabra SK, Barar V, Bansal RK, et al. Upper and lower limb standards in newborn. Indian Pediatr 1989;26:667-70.

21 Kulkarni ML, Rajendran NK. Values for foot length in newborns. Indian Pediatr 1992;29:507-9.

22 Mathur A, Tak SK, Kothari P. 'Foot length'--a newer approach in neonatal anthropometry. J Trop Pediatr 1984;30:333-6.

23 Singhal S, Tomar A, Masand R, et al. A simple tool for assessment of gestational age in newborns using foot length. J Evol Med Dent Sci 2014;3:6424-9.

24 Srivastava A, Sharma U, Kumar S. To study correlation of foot length and gestational age of new born by new Ballard score. Int J Res Med Sci 2015;3:3119-22.

25 Merlob P, Sivan Y, Reisner SH. Lower limb standards in newborns. Am J Dis Child 1984;138:140-2.

26 Vocel J, Marková H. [Signifivance of sole dermatoglyphics and of foot length in the accurate determination of gestational age in newborn infants]. Cesk Pediatr 1978;33:618-20.

27 Usher R, McLean F. Intrauterine growth of live-born Caucasian infants at sea level: standards obtained from measurements in 7 dimensions of infants born between 25 and 44 weeks of gestation. $J$ Pediatr 1969;74:901-10.
28 Lee AC, Mullany LC, Ladhani K, et al. Validity of newborn clinical assessment to determine gestational age in Bangladesh. Pediatrics 2016;138:peds.2015-3303.

29 Kc A, Nelin V, Vitrakoti R, et al. Validation of the foot length measure as an alternative tool to identify low birth weight and preterm babies in a low-resource setting like Nepal: a cross-sectional study. BMC Pediatr 2015;15:43.

30 Pratinidhi AK, Bagade AC, Kakade SV, et al. Action-oriented colour-coded foot length calliper for primary healthcare workers as a proxy for birth weight \& gestational period. Indian J Med Res 2017;145:347-52.

31 Roy RA, Rao SS, Mithra P. Correlation of foot length and gestational maturity in Neonates-A study from coastal Karnataka. Indian J Public Health Res Dev 2019;10:223-35.

32 Nabiwemba E, Marchant T, Namazzi G, et al. Identifying high-risk babies born in the community using foot length measurement at birth in Uganda. Child Care Health Dev 2013a;39:20-6.

33 Paulsen CB, Nielsen BB, Msemo OA, et al. Anthropometric measurements can identify small for gestational age newborns: a cohort study in rural Tanzania. BMC Pediatr 2019;19:120.

34 Gidi NW, Berhane M, Girma T, et al. Anthropometric measures that identify premature and low birth weight newborns in Ethiopia: a cross-sectional study with community follow-up. Arch Dis Child 2020;105:326-31.

35 Mukherjee S, Roy P, Mitra S, et al. Measuring new born foot length to identify small babies in need of extra care: a cross-sectional hospital based study. Iran J Pediatr 2013;23:508-12.

36 Srinivasa S, Manasa G, Madhu GN. Foot length of newborn: its correlation with gestational age and various anthropometric parameters. Curr Pediatr Res 2017;21:248-53.

37 Marchant T, Jaribu J, Penfold S, et al. Measuring newborn foot length to identify small babies in need of extra care: a cross sectional hospital based study with community follow-up in Tanzania. BMC Public Health 2010;10:624.

38 Otupiri E, Wobil P, Nguah SB, et al. Anthropometric measurements: options for identifying low birth weight newborns in Kumasi, Ghana. PLoS One 2014:9:e106712.

39 Hadush MY, Berhe AH, Medhanyie AA. Foot length, chest and head circumference measurements in detection of low birth weight neonates in Mekelle, Ethiopia: a hospital based cross sectional study. BMC Pediatr 2017;17:111.

40 PAS. Foot length and surrogate neonatal Anthropometrics to identify preterm and low birth weight infants in rural Bangladesh. San Francisco, CA.: Pediatric Academic Societies (PAS) Annual Meeting, 2017.

41 Gueye M, Sylla A, Diagne R, et al. Screening of low-birthweight babies with foot length measurement. Archives de Pediatrie 2014;21.

42 Gohil JR, Sosi M, Vani SN, et al. Footlength measurement in the neonate. Indian J Pediatr 1991;58:675-7.

43 Daga SR, Daga AS, Patole S, et al. Foot length measurement from foot print for identifying a newborn at risk. J Trop Pediat 1988;34:16-19.

44 Hirve SS, Ganatra BR. Foot tape measure for identification of low birth weight newborns. Indian Pediatr 1993;30:25-9.

45 Elizabeth NL, Christopher OG, Patrick K. Determining an anthropometric surrogate measure for identifying low birth weight babies in Uganda: a hospital-based cross sectional study. BMC Pediatr 2013b;13:54.

46 Marchant T, Penfold S, Mkumbo E, et al. The reliability of a newborn foot length measurement tool used by community volunteers to identify low birth weight or premature babies born at home in southern Tanzania. BMC Public Health 2014;14:859.

47 James DK, Dryburgh EH, Chiswick ML. Foot length--a new and potentially useful measurement in the neonate. Arch Dis Child 1979;54:226-30

48 Sateesha SR, Niranjan HS, Jagadish AS, et al. Correlation of Foot \& Hand Length Measurement with Gestational Maturity in Neonates. Int J Pharm Bio Sci 2015;6:1050-4.

49 Lee AC, Panchal P, Folger L, et al. Diagnostic accuracy of neonata assessment for gestational age determination: a systematic review. Pediatrics 2017;140:e20171423.

50 Eregie CO. A new method for maturity determination in newborn infants. J Trop Pediatr 2000;46:140-4

51 Lee AC, Kozuki N, Cousens S, et al. Estimates of burden and consequences of infants born small for gestational age in low and middle income countries with INTERGROWTH-2 $1^{\text {st }}$ standard: analysis of CHERG datasets. BMJ 2017;358:j3677.

52 Hodgins S, Rajbhandari B, Joshi D, et al. Community-Based cluster randomized controlled trial: empowering households to identify and provide appropriate care for low-birthweight newborns in Nepal. BMC Public Health 2020;20:1274. 